\title{
Incidence and Case Fatality at the County Level as Contributors to Geographic Disparities in Stroke Mortality
}

\author{
Darwin R. Labarthe ${ }^{a}$ George Howard ${ }^{b}$ Monika M. Safford ${ }^{d}$ Virginia J. Howard ${ }^{c}$ \\ Suzanne E. Judd ${ }^{b}$ Mary Cushman ${ }^{\text {e }}$ Brett M. Kissela ${ }^{f}$ for the Reasons for \\ Geographic and Racial Differences in Stroke (REGARDS) Investigators \\ ${ }^{a}$ Department of Preventive Medicine, Northwestern University Feinberg School of Medicine, Chicago, III., ${ }^{\text {b Department }}$ \\ of Biostatistics and 'Department of Epidemiology, School of Public Health, University of Alabama at Birmingham, \\ Birmingham, Ala., dDepartment of General Internal Medicine, Weill Cornell School of Medicine, New York, N.Y., \\ e Department of Medicine, School of Medicine, University of Vermont, Burlington, Vt., and ${ }^{\mathrm{f}}$ Department of Neurology, \\ School of Medicine, University of Cincinnati, Cincinnati, Ohio, USA
}

\section{Key Words}

Stroke $\cdot$ Geography $\cdot$ Mortality $\cdot$ Incidence $\cdot$ Case fatality

\begin{abstract}
Background: Is the high stroke mortality in the Southeastern parts of the United States driven by differences in stroke incidence or case-fatality? This question remains unanswered. Differences in incidence would underscore the need for stroke prevention, while differences in case fatality would call for improved stroke care. Methods: Quartiles of US counties were defined by stroke mortality, and this gradient was related with stroke incidence and stroke case fatality in the Reasons for Geographic and Racial Differences in Stroke (REGARDS) Study, where 1,317 incident stroke events (of which 242 were fatal) occurred among 29,650 participants. Results: There was a significant $(p=0.0025)$ gradient of fatal stroke events in REGARDS (quartile 4 vs. quartile 1 (Q4/Q1) hazard ratio $1.95,95 \% \mathrm{Cl} 1.35-2.81$ ), demonstrating the consistency of REGARDS with national mortality data. The gradient for incident stroke (fatal + nonfatal) was also significant
\end{abstract}

\section{KARGER}

(C) 2016 S. Karger AG, Basel

E-Mail karger@karger.com

www.karger.com/ned $(\mathrm{p}=0.0023 ; \mathrm{Q} 4 / \mathrm{Q} 1$ hazard ratio $1.29,95 \% \mathrm{Cl} 1.10-1.52)$. The gradient for stroke case-fatality was marginally significant $(p=0.058)$, though the OR for Q4/Q1 (1.71, 95\% Cl 1.13-2.25) was large. Conclusions: Both stroke incidence and case-fatality in REGARDS appear to be contributing, underscoring the need for strengthening both stroke prevention and acute stroke care in order to reduce the disparity.

(c) 2016 S. Karger AG, Basel

\section{Introduction}

There has been awareness about geographic variation in stroke mortality between US counties for more than half a century now $[1,2]$, with a 'stroke belt' region of exceptionally high mortality rates generally defined as comprising 8 the southeastern states: North Carolina, South Carolina, Georgia, Tennessee, Alabama, Mississippi, Arkansas, and Louisiana [3-5]. The 'stroke buckle' is a still more concentrated aggregation of high mortality-rate counties in the eastern, coastal plains of 3 of these states - 
North Carolina, South Carolina, and Georgia [6]. The high stroke-risk counties in the stroke belt exhibit 3-4 times the rate of death from stroke seen in low-risk counties from other regions [7]. While these differences are smaller than the approximate, tenfold difference in stroke mortality between nations, they are striking in that they occur within a much smaller geographic area despite presumably narrower differences in social and environmental conditions [8-10].

Death rates from stroke, as for other causes, are the product of 2 components - the rate of disease events (incidence) and the proportion of these events resulting in death (case fatality), that is, mortality $=$ incidence $\times$ case fatality. The question whether incidence or case fatality is contributing to the geographic disparity has significant implications: (1) if variation in incidence is the driving factor, then reducing the geographic disparity requires population-based preventive interventions to reduce the higher stroke event rates in the high mortality regions; while (2) if variation in case fatality is the driving factor, then modification of the health care system serving acute stroke victims must be addressed.

Because the United States lacks a national surveillance system capable of providing data on stroke incidence and case fatality, data have been unavailable on how either component relates to the geographic variation in stroke mortality. However, the Reasons for Geographic and Racial Differences in Stroke (REGARDS) Study provides data on a large population sample of blacks and whites across the United States and offers the opportunity to assess both stroke incidence and case fatality by the county of residence.

The heterogeneity in stroke mortality over relatively short distances implies that analysis by areas as large as states would tend to obscure true variation. Analysis at the county level allows the required precision in defining the areas to be compared and assuring sensitivity to detect geographic associations between factors of interest and occurrence of stroke events.

\section{Methods}

We first assessed whether geographic patterns of overall stroke deaths in the REGARDS Study parallel those reported in US Vital Statistics, including all types and both first and recurrent stroke events. If so, this would demonstrate consistency between the REGARDS Study and the US Vital Statistics information. Subsequently, we addressed our central question with respect to geographic variation: whether stroke incidence, case fatality, or both, among REGARDS Study participants are predicted by the gradient of county-level US stroke mortality.

Causes of the Geographic Disparity in Stroke Mortality
The Predictor Variable: County-Level Stroke Mortality, US

Cerebrovascular disease mortality (inclusive of ICD10: I60I69) at the county level is derived from stroke deaths as reported by the NCHS [11]. Validity of ICD10 codes in this category has been reported as $\geq 82 \%$ sensitive and $\geq 95 \%$ specific [12]. Because counties with small populations have highly unstable estimates of stroke mortality, NCHS has provided 'smoothed' race-specific, county-level rates for the years 2005-2007 and 2008-2010, for persons aged 35 years and over [13].

From these data, quartiles of US stroke mortality were developed separately for blacks and whites because (1) stroke mortality is dramatically higher for blacks than whites; (2) the pattern of geographic disparity in stroke mortality differs slightly for blacks and whites; and (3) there are a substantial number of counties with an insufficient black population to provide stable estimates. NCHS excludes estimates from counties with either less than 100 population-years of exposure or fewer than 20 events within the subject and adjacent counties, and we deleted such counties from analysis. Of the 3,109 counties in the continental United States, 27 were excluded for whites and 1,562 for blacks, thereby making analysis possible in 3,082 and 1,547 counties, respectively. We then averaged the 2 smoothed race-specific NCHS estimates for these counties and calculated race-specific quartiles. The geographic distributions of these rates, with their corresponding race-specific quartile ranges, are mapped in figure 1. For whites, the median mortality/100,000/year within each quartile was $72.8,83.5,92.2$, and 106.3 (a $46 \%$ increase across the range); for blacks, the median mortality within each quartile was $105.7,122.4,138.8$, and 162.6 (a 54\% increase across the range and approximately $50 \%$ greater for blacks than whites in each quartile).

The Dependent Variables: Stroke Death, Stroke Incidence, and Stroke Case Fatality, REGARDS Study

The source of data for partitioning stroke death rates into its components of incidence and case fatality was the REGARDS Study, a community-based, longitudinal cohort study of 30,239 black and white individuals, recruited between 2003 and 2007. Only persons aged 45 years or older were eligible, as too few strokes would be expected to occur below age 45 to add power to the study [14]. The study oversampled residents of the stroke belt/buckle (56\% of participants), with the remaining $44 \%$ of REGARDS participants drawn from residents of the other 40 contiguous US states, with participants from 1,833 US counties (approximately $60 \%$ of the counties in the continental United States). Details of the sampling approach and baseline evaluation were published previously [14]. Of the eligible participants contacted, the cooperation rate was $49 \%$. The recruitment design did not afford an opportunity to assess characteristics of individuals not included in the final sample.

For consistency with US stroke mortality statistics (which do not distinguish between deaths from first or recurrent strokes, nor between or stroke subtypes), we include all REGARDS physicianadjudicated stroke events during follow-up, including those with prior stroke at baseline, and regardless of stroke subtype.

Telephone surveillance for potential stroke events was conducted at 6-month intervals, with medical records retrieved and physician-adjudicated for all suspected strokes. All-cause mortality is ascertained through active follow-up with participants or their proxies and searches of both the Social Security Administra-

Neuroepidemiology 2016;47:96-102 


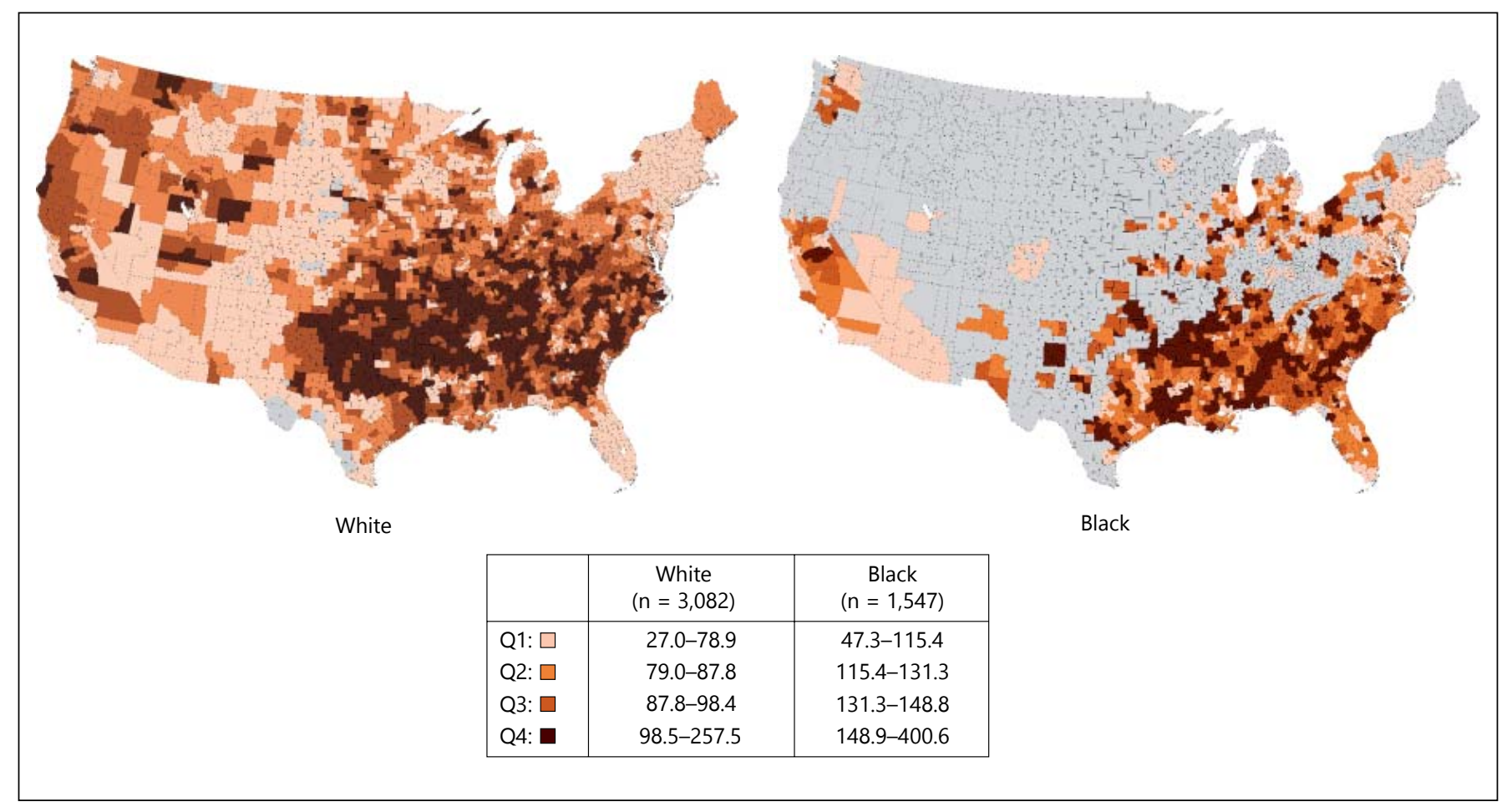

Fig. 1. County-level quartile (distribution in table) of stroke mortality age $35+$ shown by race, calculated as the average value of ageadjusted rates for the years 2005-2007 and 2008-2010, for 48 contiguous states. Areas with insufficient population for reliable esti- mation are shown in gray. Adapted from Centers for Disease Control and Prevention, National Center for Health Statistics, 2012. tion's Master Death File [15] and the National Death Index [16]. We define an incident fatal stroke as death within 30 days of a physician-adjudicated stroke event. An incident stroke is defined as a physician-adjudicated fatal or nonfatal stroke detected during follow-up. Case fatality is defined as the proportion of participants who suffered an incident stroke and died within 30 days of this stroke event.

Our analysis proceeded in 3 main steps:

(1) Examination of the heterogeneity of county-specific levels of stroke mortality even within regions traditionally defined as the stroke buckle, stroke belt, and the rest of the nation.

(2) Assessment of the geographic pattern of incident fatal strokes within REGARDS by quartile of stroke mortality in US Vital Statistics. Consistency in this comparison would support the representativeness of the REGARDS Study population.

(3) Analysis of the REGARDS outcome data for incident stroke events and case fatality by quartile of county-level US stroke mortality predicts parallel gradients in stroke incidence, case fatality, or both.

Associations between US stroke mortality data and incident fatal stroke rates and all incident stroke rates in REGARDS were assessed using Poisson regression and proportional hazards analysis, while logistic regression was used for case fatality rates. Potential interactions with race were assessed by a race-by-quartile term, with an a priori plan to pool black and white populations and adjust for race if that term was nonsignificant $(p>0.10)$.

\section{Results}

Of the 30,239 REGARDS participants, we excluded 56 $(0.2 \%)$ due to data anomalies, and $11(<0.1 \%)$ for whom the county of residence could not be definitively determined. Of the remaining 30,172 , follow-up was available on 29,650 (98.3\%), who served as the basis for these analyses. As shown in table 1, the distributions of participants by sex and race were balanced across quartile groups.

Table 1 also shows that REGARDS Study participants living in quartile $4(\mathrm{Q} 4)$ counties were most often residents of stroke buckle (33.6\%) or stroke belt (51.6\%) counties and least often residents in the rest of the nation (14.8\%). As shown in figure 1, the heterogeneity of stroke mortality by county throughout the United States demonstrated both the existence of counties with low-stroke mortality in the stroke buckle/belt regions and by the existence of high-stroke risk counties in the rest of the nation.

The underlying numbers of REGARDS Study participants, incident stroke events, fatal stroke events, and person-years of exposure during follow-up are shown in ta- 
Table 1. Characteristics of the study population by quartile of county-level stroke mortality as calculated from US Vital Statistics, 2005-2007 and 2008-2010 [10,11]

\begin{tabular}{|c|c|c|c|c|}
\hline & \multicolumn{4}{|c|}{ Quartile of county stroke mortality } \\
\hline & $\mathrm{Q} 1(\mathrm{n}=7,357)$ & $\mathrm{Q} 2(\mathrm{n}=7,468)$ & Q3 $(\mathrm{n}=7,395)$ & $\mathrm{Q} 4(\mathrm{n}=7,430)$ \\
\hline Age, years, mean $\pm S D$ & $65.4 \pm 9.4$ & $65.1 \pm 9.5$ & $64.8 \pm 9.4$ & $64.3 \pm 9.3$ \\
\hline Male, $\%$ & 47.9 & 45.3 & 43.2 & 43.3 \\
\hline Black, $\%$ & 41.7 & 40.5 & 41.2 & 41.1 \\
\hline \multicolumn{5}{|l|}{ Region of the nation, $\%$} \\
\hline Buckle & 4.9 & 16.6 & 28.5 & 33.6 \\
\hline Belt & 11.9 & 34.7 & 40.4 & 51.6 \\
\hline Rest of nation & 83.2 & 48.7 & 31.1 & 14.8 \\
\hline
\end{tabular}

Table 2. Numbers of participants, stroke events, fatal strokes, and person-years of exposure within strata of county-level stroke mortality, as calculated from US Vital Statistics, 2005-2007 and 2008-2010 [10, 11]

\begin{tabular}{lrrrrr}
\hline & \multirow{2}{*}{ All } & \multicolumn{4}{l}{ Quartile of county stroke mortality } \\
\cline { 3 - 6 } & & Q1 & Q2 & Q3 & Q4 \\
\hline Number of participants & 29,650 & 7,357 & 7,468 & 7,395 & 7,430 \\
Number of stroke events & 1,317 & 317 & 320 & 315 & 365 \\
Number of fatal stroke events & 242 & 46 & 68 & 41 & 77 \\
Person-years exposure & 205,917 & 52,615 & 51,913 & 50,993 & 50,396 \\
\hline
\end{tabular}

ble 2. Altogether, among 29,650 REGARDS participants, 205,917 person-years of exposure were observed, with a total of 1,317 stroke events of which 242 were fatal.

The key findings for fatal strokes, any incident stroke, and case-fatality according to quartile of US stroke mortality are shown in table 3 . We found no evidence of interaction between race and fatal stroke $(p=0.24)$, incident stroke $(p=0.82)$ or case fatality $(p=0.39)$; therefore, data for blacks and whites are pooled with adjustment for race.

For incident fatal strokes in REGARDS, a significant gradient of rates roughly parallels that of the quartiles of US stroke mortality, greater for Q4 with the hazard ratio (vs. Q1) of 1.95 (95\% CI 1.35-2.81; table 3). The rate for Q2 is greater than that for Q3; however, the test for linear trend assessing increasing risk of fatal stroke across quartiles was clearly significant ( $\mathrm{p}=0.0025)$.

For total incident stroke events (fatal + nonfatal), rates increase monotonically across quartiles of US stroke mortality, and the observed gradient is highly statistically significant $(\mathrm{p}=0.0023)$. However, the magnitude of the hazard ratio for Q4/Q1 $(1.29,95 \%$ CI 1.10-1.52) is notably less strong than that for fatal stroke (table 3 ).

For stroke case fatality (table 3 ), the gradient of ORs is irregular with a linear trend just short of significance $(\mathrm{p}=$
0.058). However, the strength of the association (Q4/Q1 OR 1.71, 95\% CI 1.13-2.59) appears to be greater than that for incident stroke.

\section{Discussion}

The documented geographic disparity of stroke death rates is attributable in part to geographic variation in both incidence and case-fatality. This finding contrasts with our recent report from REGARDS on the racial (blackwhite) disparity, which is nearly completely attributable to variation in incidence and not case-fatality [17]. The latter finding suggests that while management of acute strokes is more nearly equivalent between blacks and whites, the racial disparity in stroke death rates is driven by differences in risk prior to occurrence of a stroke.

\section{The Main Observations}

Long-recognized geographic disparities in US stroke mortality persist $[5,18]$. The search for answers has included studies of stroke prevalence [19], but because prevalence reflects both incidence and case fatality, it is uninformative for the purpose of the present analysis. A 
Table 3. Estimated rates of fatal stroke and incident stroke per 100,000 per year (95\% CI) adjusted for age group (45-64, 65-74, 75+), race, sex and the age group $\times$ race interaction; and hazard ratios compared to low-mortality counties (Q1; 95\% CI) similarly adjusted; and number of fatal strokes (death within 30-days of incident stroke) and total incident stroke events; percentages of fatal stroke events, and 95\% CIs for the percentages of fatal strokes; and ORs and 95\% CIs (relative to the low mortality counties, Q1) for fatal stroke after adjustment for age group (45-64, 65-74, 75+), race, and sex. Quartiles calculated from US vital statistics, 2005-2007 and 2008-2010 [10, 11]. Proportional hazards estimated with age as a continuous factor. p-trend estimated from the proportional hazards model

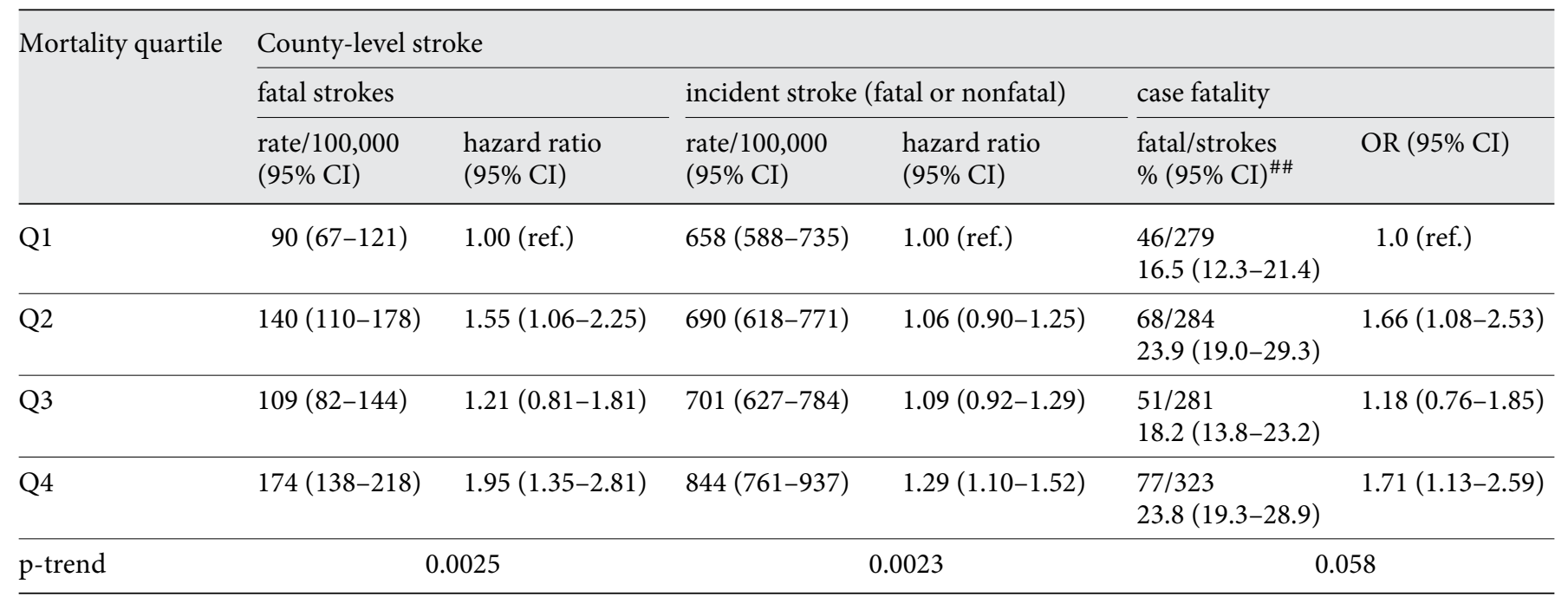

few reports have suggested that differences in incidence could be a contributing factor to the geographic disparity [20]. Further, there have been suggestions that whatever be the factors that account for the stroke belt, the impact may be larger in blacks [21]. However, in none of the 3 predictions in the present analysis - of fatal stroke, incident stroke, nor stroke case fatality - is there statistical evidence of race-by-trend interaction, supporting pooling of blacks and whites in this analysis.

The observation that the geographic pattern of fatal strokes aligns well with the Vital Statistics pattern for stroke mortality provides external validation of observations from the study. This validation offers assurance regarding the reasonable nature of the steps of addressing our central question: does incidence, case fatality, or both determine this geographic variation?

Both incidence and case fatality appear to be determinants of geographic variation in stroke mortality. Strength of association, as measured by the observed hazard ratios and ORs, somewhat favors case fatality, but the irregularity of the gradient in this association is problematic. Consistency of trends favors incidence over case fatality, given clearly statistically significant results for the first but not for the second. The present findings in the United States are congruent with previous international reports suggesting that overall temporal declines in stroke mortality reflect the joint impact of both lower incidence and reduced case-fatality [22-24].

This is a potentially fruitful, though not simple, answer to the question. Incidence as a determinant would be expected to affect stroke risk chiefly through environmental exposures, and reduction of the geographic disparity would require focused efforts to prevent stroke especially in the regions of highest stroke incidence. This suggests further search for county-level characteristics that could be the sources of such exposures, and for effective community- and population-based interventions, potentially preventing the development of stroke risk factors or improving their control. Case fatality as a determinant would be expected to reflect stroke severity or acute stroke care, suggesting a need for intervention in the health care system to improve stroke outcomes especially in regions with highest case fatality.

These interpretations require qualification in light of the strengths and limitations of the REGARDS Study. The composition of the REGARDS Study population is specifically designed for investigating the geographic disparity in stroke mortality. Rigorous methods of case ascertainment and diagnostic adjudication provide assurance of the quality of the REGARDS data, and we have already accrued more than 1,300 stroke events, supporting the analysis reported here. Among its other strengths, 
REGARDS is one of few studies with truly wide geographic heterogeneity among its participants and with precision to document true trends across quartiles of mortality rates. The data for US stroke mortality at the county level were less precise, resulting in less stability of quartile-level estimates and an expected departure from a strictly monotonic trend. Therefore, our a priori approach for testing for association was to test overall linear trends, which in effect average the patterns across quartiles. Thus, they reflect our hypothesized general increase in stroke events (incidence or case-fatality) in counties with higher Vital Statistics stroke mortality.

A key limitation lies in the numbers of fatal strokes observed to date: larger experience, with further followup of the aging REGARDS cohort, is expected to afford still greater statistical precision, possibly strengthening results that are only marginally nonsignificant in the present analysis. Further insight to both community factors determining stroke risk and health system factors affecting outcomes of acute stroke will be needed to achieve an increased impact of effective interventions.

Also, with a cooperation rate of $49 \%$, it is possible that the study population may not be strictly representative of the general population; however, the geographic pattern of fatal stroke in the cohort and in the Vital Statistics data is reassuring on this point. Finally, while within the REGARDS study population strokes are classified by subtype, this is not true for approximately half of the stroke deaths recorded in the Vital Statistics system, which are coded as I64: stroke, not specified as hemorrhage or infarction. This limitation, common to many large stroke databases, precludes fully assessing agree- ment between REGARDS and US Vital Statistics as to stroke subtypes.

The REGARDS Study experience to date suggests that both stroke incidence and stroke case fatality contribute to the persisting geographic disparities in stroke mortality. To eliminate these disparities, intensified research is warranted on both components of stroke mortality, with a dual focus on primordial or primary stroke prevention to reduce the impact of higher stroke incidence in the high-stroke mortality regions, plus ensure improvements in health care once a stroke has occurred to reduce the impact of higher case-fatality in the high-stroke mortality regions. Focusing solely on either is unlikely to eliminate the disparity.

\section{Acknowledgments}

The authors thank the investigators, staff, and participants of the REGARDS Study for their valuable contributions. A full list of participating REGARDS investigators and institutions can be found at http://www.regardsstudy.org.

\section{Sources of Funding}

This research project was supported by cooperative agreement U01-NS041588 from the National Institute of Neurological Disorders and Stroke, NIH.

\section{Disclosure Statement}

The authors report no disclosures.

\section{References}

1 Stallones RA: Epidemiology of cerebrovascular disease. A review. J Chronic Dis 1965;18: 859-872.

2 Borhani NO: Changes and geographic distribution of mortality from cerebrovascular disease. Am J Public Health Nations Health 1965;55:673-681.

3 Howard G, Howard VJ, Katholi C, Oli MK, Huston S: Decline in US stroke mortality: an analysis of temporal patterns by sex, race, and geographic region. Stroke 2001;32:2213-2220.

4 Lanska DJ, Kuller LH: The geography of stroke mortality in the United States and the concept of a stroke belt. Stroke 1995;26:11451149.

5 Perry HM, Roccella EJ: Conference report on stroke mortality in the southeastern United States. Hypertension 1998;31:1206-1215.
6 Howard G, Anderson R, Johnson NJ, Sorlie P, Russell G, Howard VJ: Evaluation of social status as a contributing factor to the stroke belt region of the United States. Stroke 1997; 28:936-940.

7 Casper ML, Barnett E, Williams GI Jr, Halverson JA, Braham VE, Greenlund KJ: Atlas of Stroke Mortality: Racial, Ethnic, and Geographic Disparities in the United States. Atlanta, Department of Health and Human Services, Centers for Disease Control and Prevention, 2003.

8 Feigin VL, Krishnamurthi RV, Parmar P, Norrving B, Mensah GA, Bennett DA, Barker-Collo S, Moran AE, Sacco RL, Truelsen T, Davis S, Pandian JD, Naghavi M, Forouzanfar $\mathrm{MH}$, Nguyen G, Johnson CO, Vos T, Meretoja A, Murray CJ, Roth GA; GBD 2013 Writ- ing Group; GBD 2013 Stroke Panel Experts Group: Update on the global burden of ischemic and hemorrhagic stroke in 1990-2013. the GBD 2013 Study. Neuroepidemiology 2015;45:161-176.

9 Feigin VL, Mensah GA, Norrving B, Murray CJ, Roth GA: Atlas of the global burden of stroke (1990-2013): the GBD 2013 study. Neuroepidemiology 2015;45:230-236.

10 Krishnamurthi RV, Moran AE, Feigin VL, Barker-Collo S, Norrving B, Mensah GA, Taylor S, Naghavi M, Forouzanfar $\mathrm{MH}$, Nguyen G, Johnson CO, Vos T, Murray CJ, Roth GA: Stroke prevalence, mortality and disability-adjusted life years in adults aged 20-64 years in 1990-2013: data from the global burden of disease 2013 study. Neuroepidemiology 2015;45:190-202. 
11 Moriyama IM, Loy RM, Robb-Smith AHT: History of the Statistical Classification of Diseases and Causes of Death. Hyattsville, National Center for Health Statistics, 2011.

12 McCormick N, Bhole V, Lacaille D, AvinaZubieta JA: Validity of diagnostic codes for acute stroke in administrative databases: a systematic review. PLoS One 2015;10: e0135834.

13 Control Centers for Disease: Interactive Atlas of Heart Disease and Stroke Tables. 2012. http://apps.nccd.cdc.gov/DHDSPAtlas/ reports.aspx.

14 Howard VJ, Cushman M, Pulley L, Gomez CR, Go RC, Prineas RJ, Graham A, Moy CS, Howard G: The reasons for geographic and racial differences in stroke study: objectives and design. Neuroepidemiology 2005;25: 135-143.

15 Schisterman EF, Whitcomb BW: Use of the social security administration death master file for ascertainment of mortality status. Popul Health Metr 2004;2:2.
16 Bilgrad R: National Death Index User's Manual. Hyattsville, US Department of Health and Human Services, PHS, Centers for Disease Control, National Center for Health Statistics, 1997.

17 Howard G, Moy CS, Howard VJ, McClure LA, Kleindorfer DO, Kissela BM, Judd SE, Unverzagt FW, Soliman EZ, Safford MM, Cushman M, Flaherty ML, Wadley VG: Where should interventions be focused to reduce the black-white disparity in stroke mortality? An assessment of black-white differences in stroke incidence and stroke case-fatality. Stroke 2016, in press.

18 Howard G: Why do we have a stroke belt in the southeastern United States? A review of unlikely and uninvestigated potential causes. Am J Med Sci 1999;317:160-167.

19 Centers for Disease Control and Prevention (CDC): Regional and racial differences in prevalence of stroke - 23 states and district of Columbia, 2003. MMWR Morb Mortal Wkly Rep 2005;54:481-484.

20 Howard VJ, Kleindorfer DO, Judd SE, McClure LA, Safford MM, Rhodes JD, Cushman M, Moy CS, Soliman EZ, Kissela BM, Howard G: Disparities in stroke incidence contributing to disparities in stroke mortality. Ann Neurol 2011;69:619-627.
21 Howard G, Labarthe DR, Hu J, Yoon S, Howard VJ: Regional differences in African Americans' high risk for stroke: the remarkable burden of stroke for Southern African Americans. Ann Epidemiol 2007;17:689-696.

22 Sarti C, Stegmayr B, Tolonen H, Mahonen M, Tuomilehto J, Asplund K; WHO MONICA Project: Are changes in mortality from stroke caused by changes in stroke event rates or case fatality? Results from the WHO MONICA project. Stroke 2003;34:1833-1840.

23 Sivenius J, Tuomilehto J, Immonen-Raiha P, Kaarisalo M, Sarti C, Torppa J, Kuulasmaa K, Mahonen M, Lehtonen A, Salomaa V; FINSTROKE Study: Continuous 15 -year decrease in incidence and mortality of stroke in Finland: the FINSTROKE study. Stroke 2004; 35:420-425.

24 Vangen-Lonne AM, Wilsgaard T, Johnsen SH, Carlsson M, Mathiesen EB: Time trends in incidence and case fatality of ischemic stroke: the Tromsø study 1977-2010. Stroke 2015;46:1173-1179. 